\title{
Matter from geometry
}

\section{Citation}

Katz, Sheldon, and Cumrun Vafa. 1997. "Matter from Geometry." Nuclear Physics B 497 (1-2): 146-54. https://doi.org/10.1016/s0550-3213(97)00280-0.

\section{Permanent link}

http://nrs.harvard.edu/urn-3:HUL.InstRepos:41385075

\section{Terms of Use}

This article was downloaded from Harvard University's DASH repository, and is made available under the terms and conditions applicable to Other Posted Material, as set forth at http:// nrs.harvard.edu/urn-3:HUL.InstRepos:dash.current.terms-of-use\#LAA

\section{Share Your Story}

The Harvard community has made this article openly available.

Please share how this access benefits you. Submit a story.

Accessibility 
hep-th/9606086, HUTP-96/A021,OSU-M-96-10

\title{
Matter From Geometry
}

\author{
Sheldon Katz \\ Department of Mathematics \\ Oklahoma State University \\ Stillwater, OK 74078, USA \\ and \\ Cumrun Vafa \\ Lyman Laboratory of Physics \\ Harvard University \\ Cambridge, MA 02138, USA
}

We provide a local geometric description of how charged matter arises in type IIA, M-theory, or F-theory compactifications on Calabi-Yau manifolds. The basic idea is to deform a higher singularity into a lower one through Cartan deformations which vary over space. The results agree with expectations based on string dualities.

6/96 


\section{Introduction}

In a recent work [1] a detailed check was made of the enhanced gauge symmetry points of F-theory/heterotic duality in $d=6$ with $N=1$ supersymmetry proposed in [2] . There it was shown how charged matter should arise upon compactification of F-theory to a Calabi-Yau threefold for consistency with F-theory/heterotic duality. As far as the matter content is concerned various results were found in [1] based on the duality with heterotic strings [2] but a purely F-theory (type IIA) derivation for the matter content was lacking. In particular it was observed there that for simply laced gauge groups very often the matter seems localized at 'extra' singularities of the manifold. Here we wish to derive this structure from the viewpoint of F-theory alone thus sharpening the F-theory/heterotic duality check. A heterotic derivation of this localization has been recently done by Witten [3]. In fact the physical interpretation of the localization we find is identical to that found there.

We will recover not only the matter structure/geometry dictionary anticipated in [1] but also present examples of new such cases. Our aim here is not to be exhaustive in this matter/geometry dictionary but only to present the main idea through examples.

\section{Basic Idea}

We first consider compactification of F-theory to 8 dimensions (or type IIA to 6 dimensions) on an elliptic (general) $K 3$ 四. Suppose we are at a moduli value of $K 3$ where we have a singularity of A-D-E type. In this case the gauge symmetry is the corresponding A-D-E group. We consider a further compactification of this theory to lower dimensions. For concreteness let us consider further compactification on a one dimensional complex space denoted by a parameter $t$, where the moduli of $K 3$ is varying over this space. We wish to find the gauge group and matter representation in the lower dimensional theory. The gauge group is easy to identify simply by considering what is the singularity type over generic $t$ [5] [1]; there could also be further monodromy acting on the singularity leading to non-simply laced groups [5]. Let us concentrate on the case where there are no further monodromies, i.e. what is called the 'split' case though our results can be partially generalized to the other case as well.

To fix our terminology, let $G$ denote the corresponding A-D-E group. There is a complex scalar field in the adjoint representation of $G$. For a generic expectation value of this scalar the group $G$ is broken to $U(1)^{n}$ where $n$ is the rank of $G$. For special $U(1)$ 's we 
can have $H \times U(1) \subset G$ where $H$ has no $U(1)$ factors and has rank one lower than that of $G$. The basic idea is to consider fiberings where the scalar field in this $U(1)$ direction is identified with the fibration parameter $t$. Thus the surviving gauge symmetry in the lower dimensional theory is $H$. The massless matter representation for $H$ can be read off by decomposing the matter which is in the adjoint representation of $G$ in terms of $H \times U(1)$ representations and by finding the zero modes of the Dirac operator coupled to the gauge bundle given by $U(1)$. Let $\left(R^{a}, q^{a}\right)$ denote the representations we get where $q^{a}$ denote the $U(1)$ charge. If the fibration were trivial the gauge symmetry would have been $G$ and the matter would have been in the adjoint representation of $G$. This is still true at one point on the fibration $t=0$ where $G$ symmetry is restored. So we still will get the adjoint representation of $G$, but the effect of fibration is to make that matter a representation of $H$ according to how the adjoint decomposes. To see this let $t$ denote the complexified Cartan of the $U(1)$, which we identify with the fibering parameter. Then the Dirac operator giving the number of zero modes of $R^{a}$ is given by solving

$$
\left[D+q_{a} t\right] \psi_{a}(t, \bar{t})=0
$$

where $D$ denotes the Dirac operator on $t$-plane. The number of $R^{a}$ representations we will get is given by the number of normalizable zero modes of (2.1) which in turn is one (if $q_{a} \neq 0$ )-note that the zero mode is localized near $t=0$ which after a suitable rescaling of $t$ goes as $\propto \exp (-t \bar{t}))^{1}$. Here we are concentrating on the matter localized at the 'extra' singularities and so our considerations are all local with respect to the $t$-parameter; we are not concerned with other matter which are not concentrated at these extra singularities 2 . There are cases where the matter is not localized in this way. For example, in the type IIA theory when we have a genus $g$ curve of singularities of $A_{n}$ type we get $g$ adjoints of $A_{n}$ [6] which are not localized on any specific points on the curve.

As far as the description of the matter is concerned we can give two alternative descriptions, depending on whether we are talking about F-theory down to six dimensions or type IIA down to 4 . For F-theory description, let us assume that $G=S U(n)$, where the geometry is realized by $n$ coinciding 7-branes. Then as a function of $t$ the location of

1 This idea is well known in the context of family's index theorem applied to the case at hand where $t$ is the parameter space and the localization of the zero modes occurs at some points on the parameter space.

2 This is why the representations with $q_{a}=0$ are not relevant for our considerations. 
the 7-branes is changing and the resulting open strings pick up mass as a function of $t$ and we are finding the wave function of the massless modes of open strings concentrated near $t=0$. As far as the type IIA description is concerned, we have an adjoint of $G$ worth of vanishing 2-cycles, with D-branes wrapped around them. As we move away from $t=0$ some of the 2-cycles pick up mass and the wave function of the 2-branes are concentrated near $t=0$.

Note that if we replace $t \rightarrow P_{n}(t)$, where $P_{n}(t)$ is a polynomial of degree $n$ in $t$, since the above considerations are local, we learn that we obtain $n$ times the matter we get, localized near the zeroes of $P_{n}(t)$.

To use the above basic idea what one needs to know is how the expectation value of the scalar taking its value in the complexified Cartan space $\mathcal{C}$ of the A-D-E modifies and resolves the singularity. Luckily this is mathematically known.

\section{Cartan Resolution of Singularities}

As discussed above one needs to know how giving expectation values to the Cartan elements of the singularity deform (and resolve) the singularity. This is a well known mathematical result. A unified treatment is given in [7], which contains references to earlier foundational works. The deformation space of the resolved singularity can be identified with the Cartan subalgebra. Here we describe the result, which gives the conditions on these expectation values for certain curves in the resolution to deform. We do this separately for $A_{n}, D_{n}, E_{n}$. In each case, we let $\left\{e_{i}\right\}$ denote an orthonormal set of vectors.

The $A_{n}$ Cartan subalgebra $h$ is the space of vectors $\sum_{i=1}^{n+1} t_{i} e_{i}$ subject to the constraint $\sum t_{i}=0$. The deformation space of the resolution of the $A_{n}$ singularity similarly has coordinates $\left(t_{1}, \ldots, t_{n+1}\right)$ subject to $\sum t_{i}=0$.

The roots are in the dual space $h^{*}$, and are all of the form $e_{i}^{*}-e_{j}^{*}$ for $i \neq j$. The simple roots are $v_{i}=e_{i}^{*}-e_{i+1}^{*}$. Each $v_{i}$ corresponds to a vertex of the Dynkin diagram and to a curve $C_{i}$ in the resolution of the $A_{n}$ singularity. To each positive root $e_{i}^{*}-e_{j}^{*}$ (so that $i<j$ ), there is associated a set of vanishing 2-cycles $C_{i j}=C_{i}+\ldots+C_{j-1}$, and the condition on the $t_{k}$ for $C_{i j}$ to remain a vanishing 2 -cycle is that $t_{i}-t_{j}=0$. The deformation of the $A_{n}$ singularity when we deform by expectation values in the Cartan is given by

$$
x y+\prod_{j=1}^{n+1}\left(z+t_{j}\right)=0 .
$$


The $D_{n}$ Cartan subalgebra is the space of vectors $\sum_{i=1}^{n} t_{i} e_{i}$. The deformation space of the resolution of the $D_{n}$ singularity has coordinates $t_{1}, \ldots, t_{n}$. The simple roots are $v_{i}=e_{i}^{*}-e_{i+1}^{*}$ for $1 \leq i \leq n-1$, and $v_{n}=e_{n-1}^{*}+e_{n}^{*}$. Each $v_{i}$ corresponds to a vertex of the Dynkin diagram and to a curve $C_{i}$ in the resolution of the $D_{n}$ singularity. The positive roots are all of the form $e_{i}^{*} \pm e_{j}^{*}$. In terms of the $v_{i}$, the positive roots are of one of the five forms $v_{i}+\ldots+v_{j-1}, v_{n}, v_{j}+\ldots+v_{n-2}+v_{n}, v_{j}+\ldots+v_{n}$, $v_{j}+\ldots+v_{k-1}+2 v_{k}+\ldots+2 v_{n-2}+v_{n-1}+v_{n}$. To each positive root $r$ is associated a curve $C$ by substituting $C_{i}$ for $v_{i}$ in the above expressions of the root. The $D_{n}$ singularity deformed by the Cartan parameters is given as

$$
x^{2}+y^{2} z-\frac{\prod_{i=1}^{n}\left(z+t_{i}^{2}\right)-\prod_{i=1}^{n} t_{i}^{2}}{z}+2 y \prod_{i} t_{i}=0 .
$$

The $E_{n}$ Cartan subalgebra is the space of vectors $\sum_{i=0}^{n} a_{i} e_{i}$ subject to the constraint $-3 a_{0}+\sum_{i=1}^{n} a_{i}=0$. The deformation space of the resolution of the $E_{n}$ singularity can be assigned coordinates $t_{1}, \ldots, t_{n}$, where $t_{i}=a_{0} / 3+a_{i}$. The simple roots are $v_{0}=$ $e_{0}^{*}-e_{1}^{*}-e_{2}^{*}-e_{3}^{*}$, and $v_{i}=e_{i}^{*}-e_{i+1}^{*}$ for $1 \leq i \leq n-1$. Each $v_{i}$ corresponds to a vertex of the Dynkin diagram and to a curve $C_{i}$ in the resolution of the $E_{n}$ singularity. The deformation of $E_{n}$ singularities in terms of Cartan parameters is more complicated. The coefficients of the $E_{6}$ and $E_{7}$ deformed polynomials are given in [7], Appendices 1 and 2. The $E_{8}$ case is known implicitly [7].

\section{The Cases}

As mentioned in the introduction the aim is to illustrate how local geometric singularity encodes charged matter, and not so much to provide an exhaustive matter/geometry dictionary. In this spirit we provide some illustrative examples below. It is possible to check in all the cases below that have an overlap with results based on F-theory/heterotic duality studied in [1], the local structure of the singularity is in agreement with expectations (there is a change of notation relative to [1] where there roughly is an interchange in what we denote as $x, y$ ). However, in some cases (with real representations) we obtain one hypermultiplet corresponding to appearance of the deformation by $t^{2}$ in the singularity. This is consistent with the results of [1] where the appearance of $t$ in the singularity was associated with one half a hypermultiplet in the real representation. 
4.1. $A_{n} \rightarrow A_{n-k} \times A_{k-1}$

We consider the breaking

$$
S U(k) \times S U(n-k+1) \times U(1) \subset S U(n+1)
$$

In this case by the decomposition of the adjoint of $A_{n}$ we expect the charged matter to be in the $(\mathbf{k}, \mathbf{n}-\mathbf{k}+\mathbf{1})$ representation of $S U(k) \times S U(n-k+1)$.

The above breaking of $A_{n}$ can be done by choosing the $U(1)$ Cartan to correspond to the $t$ direction given by

$$
\begin{gathered}
t_{1}-t_{2}=\ldots=t_{k-1}-t_{k}=t_{k+1}-t_{k+2}=\ldots t_{n}-t_{n+1}=0 \\
t_{n}-t_{1}=t .
\end{gathered}
$$

(up to an irrelevant shift this is of the form $(0, \ldots, t, \ldots, t)$ with $k$ zeroes and $n-k+1$ $t$ 's). After this shift, the equation (3.1) becomes

$$
x y+z^{k}(z+t)^{n-k+1}=0,
$$

which for $t \neq 0$ visibly has an $A_{k-1}$ singularity at $(0,0,0)$ and an $A_{n-k}$ singularity at $(0,0,-t)$. The vanishing cycles over $t$ are now given by the curves $C_{1}, \ldots, C_{k-1}, C_{k+1}, \ldots, C_{n}$ as $t$ varies. These naturally decompose into two connected components, with $E_{1}, \ldots, E_{k-1}$ in the first component corresponding to the $S U(k)$ gauge symmetry and $E_{k+1}, \ldots, E_{n}$ in the second component corresponding to $S U(n-k+1)$ gauge symmetry. Note that by a change of coordinates, defining $z^{\prime}=z+t$ the above singularity takes the form $x y+z^{k} z^{\prime n-k+1}=0$ which was considered in [1] where it was noted that based on the Dbrane analysis of [8] (applied to the intersecting 7-branes in this case) one expects matter in $(\mathbf{k}, \mathbf{n}-\mathbf{k}+\mathbf{1})$, in agreement with the above result.

\section{2. $D_{n} \rightarrow D_{n-1}, A_{n-1}, D_{n-r} \times A_{r-1}$}

We will consider three cases corresponding to the breaking patterns:

$$
\begin{gathered}
\text { i) } S O(2 n-2) \times S O(2) \subset S O(2 n) \\
\text { ii) } S U(n) \times U(1) \subset S O(2 n) \\
\text { iii) } S O(2 n-2 r) \times S U(r) \times U(1) \subset S O(2 n)
\end{gathered}
$$


In case $i$ ) by the decomposition of adjoint we expect one hypermultiplet in the fundamental $\mathbf{2 n}-\mathbf{2}$ of $S O(2 n-2)$. In the case $i i)$ we expect one matter hypermultiplet in the antisymmetric tensor representation $\mathbf{n}(\mathbf{n}-\mathbf{1}) / \mathbf{2}$ of $S U(n)$. In the case $i i i)$ we expect one hypermultiplet in the $(\mathbf{2 n}-\mathbf{2 r}, \mathbf{r})$ of $S O(2 n-2 r) \times S U(r)$ as well as one hypermultiplet in the $(\mathbf{1}, \mathbf{r}(\mathbf{r}-\mathbf{1}) / \mathbf{2})$.

The first breaking pattern $i)$ corresponds to choosing $\left(t_{1}, \ldots, t_{n}\right)=\left(t, 0^{n-1}\right)$. Plugging this into equation (3.2) we find

$$
x y+y^{2} z-z^{n-1}-t^{2} z^{n-2}=0 .
$$

We see the $D_{n}$ for $t=0$ but a $D_{n-1}$ for $t \neq 0$.

The symmetry breaking $i i)$ corresponds to choosing $\left(t_{1}, \ldots, t_{n}\right)=(t, t, \ldots, t)$. Plugging this into equation $(3.2)$ gives

$$
x^{2}+y^{2} z-\frac{\left(z+t^{2}\right)^{n}-t^{2 n}}{z}+2 t^{n} y=0
$$

which is $D_{n}$ for $t=0$ and $A_{n-1}$ for $t \neq 0$ as we now argue. There is a singularity at $\left(0, t^{n-2},-t^{2}\right)$. Accordingly, we note that near $z=-t^{2}$, this equation has leading behavior

$$
x^{2}-t^{2}\left(y-t^{n-2} z\right)^{2}+\frac{\left(z+t^{2}\right)^{n}}{t^{2}}=0
$$

which shows that we have an $A_{n-1}$ singularity for $t \neq 0$.

Note that in [1] it was found that, unlike the $A_{n}$ case, one does not seem to get intersecting $D_{n}$ singularities corresponding to mixed matter representations. This is actually explained in our case by noting that there is no $U(1)$ which breaks the $D_{n}$ to the product of two D's. The closest that we come to a product structure is the case iii) above, where we take $\left(t_{1}, \ldots, t_{r}, t_{r+1}, \ldots, t_{n}\right)=(t, \ldots, t, 0, \ldots, 0)$. Plugging this into (3.2) gives

$$
x^{2}+y^{2} z-z^{n-r-1}\left(z+t^{2}\right)^{r}=0 .
$$

Note that this is visibly a $D_{n}$ for $t=0$; for $t \neq 0$ we have a product singularity, where at $(x, y, z)=(0,0,0)$ we have $D_{n-r}$ singularity and at $(x, y, z)=\left(0,0,-t^{2}\right)$ we have an $A_{r-1}$ singularity.

\section{3. $E_{n}$ cases}

In this section we consider some breakings starting from the exceptional groups $E_{6}, E_{7}$ and $E_{8}$. These are somewhat more challenging because as mentioned before the Cartan deformations of the singularity are more complicated. 


\section{4. $E_{6} \rightarrow D_{5}, A_{5}$}

We consider two examples for the $E_{6}$ case corresponding to the decomposition

$$
\begin{aligned}
& \text { i) } S O(10) \times U(1) \subset E_{6} \\
& \text { ii) } S U(6) \times U(1) \subset E_{6}
\end{aligned}
$$

In the case $i$ ) by the adjoint decomposition we expect a localized matter in the $\mathbf{1 6}$ of $S O(10)$, and for case $i$ ) we expect matter in the $\mathbf{2 0}$ of $S U(6)$ (third rank anti-symmetric tensor representation).

The case $i$ ) occurs when $\left(t_{1}, \ldots, t_{6}\right)=(t,-2 t, t, t, t, t)$, from which the equation for the singularity can be derived to be

$$
-x^{2}+\frac{z^{4}}{4}+y^{3}-3 t^{2} y z^{2}-12 t^{5} y z-6 z^{2} t^{6}-12 t^{8} y-16 t^{9} z-12 t^{12}=0 .
$$

Shifting the singularity at $\left(0,0,-2 t^{3}\right)$ to the origin, the equation becomes

$$
\begin{aligned}
& -x^{2}+\frac{z^{4}}{4}-2 z^{3} t^{3}+y^{3}-3 t^{2} y z^{2}= \\
& -x^{2}-(2 z t-y)(z t+y)^{2}+\frac{z^{4}}{4}=0,
\end{aligned}
$$

which is visibly a $D_{5}$ singularity for $t \neq 0$.

The second case occurs by choosing the $U(1)$ to correspond to $\left(t_{1}, \ldots, t_{6}\right)=$ $(-t / 2,-t / 2,-t / 2, t, t, t)$, from which the equation for the singularity can be derived to be

$$
-x^{2}+\frac{z^{4}}{4}+y^{3}-\frac{9 t^{2} y z^{2}}{8}+\frac{243 z^{2} t^{6}}{512}-\frac{2187 t^{8} y}{4096}+\frac{19683 t^{12}}{131072}=0 .
$$

Translating the singularity at $\left(0,27 t^{4} / 64,0\right)$ to the origin, we get

$$
-x^{2}+\frac{z^{4}}{4}+y^{3}+\frac{81 y^{2} t^{4}}{64}-\frac{9 t^{2} y z^{2}}{8}=0 .
$$

The terms $\frac{z^{4}}{4}-\frac{9 t^{2} y z^{2}}{8}+\frac{81 y^{2} t^{4}}{64}$ are a perfect square; the natural change of coordinates reveals that there is an $A_{5}$ singularity for $t \neq 0$. 


\section{5. $E_{7} \rightarrow D_{6}, E_{6}, A_{6}$}

We will consider three cases corresponding to the breaking patterns

$$
\begin{gathered}
\text { i) } S O(12) \times U(1) \subset E_{7} \\
\text { ii) } \quad E_{6} \times U(1) \subset E_{7} \\
\text { iii) } \quad S U(7) \times U(1) \subset E_{7}
\end{gathered}
$$

The respective representations are expected to be one hypermultiplets in $S O(12), \mathbf{3 2}$; $E_{6}, \mathbf{2 7} ; S U(7), \mathbf{3 5} \oplus \mathbf{7}$.

The first case occurs by choosing the $U(1)$ to correspond to $\left(t_{1}, \ldots, t_{7}\right)=(t,-2 t, t, t, t, t, t)$, from which the equation of the singularity can be derived to be

$$
-x^{2}-y^{3}+16 y z^{3}+36 t^{2} y^{2} z=0 .
$$

The coordinate change $z \mapsto z+y /\left(36 t^{2}\right)$ yields for $t \neq 0$

$$
2916 x^{2} t^{6}-46656 y z^{3} t^{6}-3888 z^{2} y^{2} t^{4}-108 z y^{3} t^{2}-y^{4}-104976 t^{8} y^{2} z=0,
$$

which is seen to be a $D_{6}$ after completing the square in $y$.

The second case occurs by choosing $\left(t_{1}, \ldots, t_{7}\right)=(0,0,0,0,0, t, 0)$, from which the equation for the singularity can be derived to be

$$
-x^{2}-y^{3}+16 y z^{3}+3 t^{2} y^{2} z+\frac{3 t^{6} y^{2}}{8}-\frac{9 t^{8} y z}{16}+\frac{3 t^{10} z^{2}}{16}-\frac{11 t^{12} y}{256}+\frac{9 t^{14} z}{256}+\frac{7 t^{18}}{4096}=0 .
$$

Translating the singularity at $\left(0, t^{6} / 16,-t^{4} / 16\right)$ to the origin, we get

$$
3 t^{2} y^{2} z-x^{2}-y^{3}-3 y z^{2} t^{4}+16 y z^{3}+t^{6} z^{3}=0 .
$$

The change of variables $y \mapsto y+t^{2} z$ changes this to

$$
-x^{2}-y^{3}+16 y z^{3}+16 z^{4} t^{2}=0
$$

which visibly is an $E_{6}$ singularity for $t \neq 0$.

The third case occurs by choosing $\left(t_{1}, \ldots, t_{7}\right)=(-t,-t,-t, 2 t, 2 t, 2 t, 2 t)$, from which the equation of the singularity can be derived to be

$$
-x^{2}-y^{3}+16 y z^{3}+63 t^{2} y^{2} z+\frac{5103 t^{6} y^{2}}{8}+\frac{45927 t^{8} y z}{16}+\frac{413343 t^{10} z^{2}}{16}+
$$




$$
\frac{40920957 t^{12} y}{256}-\frac{90876411 t^{14} z}{256}+\frac{35255264499 t^{18}}{4096}=0 .
$$

Translating the singularity at $\left(0,-2187 t^{6} / 16,-81 t^{4} / 16\right)$ to the origin, we get

$$
\begin{gathered}
63 t^{2} y^{2} z-x^{2}-y^{3}-243 y z^{2} t^{4}+16 y z^{3}-2187 t^{6} z^{3}+59049 t^{10} z^{2}+ \\
729 t^{6} y^{2}-13122 t^{8} y z=0 .
\end{gathered}
$$

The last three terms form a perfect square of a linear expression in $y, z$. We adapt coordinates to this linear term to see that the singularity is $A_{r}$ for some $r$. To find the value of $r$, we make a linear coordinate change takes the equation to the form form $x^{2}+y^{2}+f(y, z)=0$ where $f$ has no terms of degree 2 or less. The problem is that $f(y, z)$ contains a term $y^{2} z$ which must be eliminated. We make a change of variables $z \mapsto z+\sum_{i=2}^{k} c_{i} y^{i}$ for $k=2,3, \ldots$, and recursively solve for the $c_{i}$ in order to make the coefficient of $y^{r} z$ equal to 0 . This happens first when $r=7$, and then a $y^{8}$ term appears, showing that we have an $A_{7}$ singularity for $t \neq 0$.

\section{6. $E_{8} \rightarrow E_{7}$}

We consider the decomposition $E_{7} \times U(1) \subset E_{8}$. We expect matter in the $\mathbf{5 6}$ of $E_{7}$. This corresponds to choosing the $U(1)$ to be $\left(t_{1}, \ldots, t_{8}\right)=(t, 0, \ldots, 0)$. Following [7], Appendix 0, the singularity can be described implicitly in projective coordinates $(W, X, Y, Z)$ by the parameterization

$$
\begin{gathered}
W=x^{3}-y z^{2} \\
X=y^{8} z-3 t^{3} z^{2} y^{7}-3 x^{2} t y^{7}+2 t^{3} y^{6} x^{3}+t^{6} z^{3} y^{6}-t^{6} z y^{5} x^{3}+3 t^{2} z y^{7} x \\
Y=-t^{4} x^{3} y^{3}+t^{4} y^{4} z^{2}+x y^{5}-2 t y^{5} z+t^{2} x^{2} y^{4} \\
Z=y^{3}-t^{2} x y^{2} .
\end{gathered}
$$

These satisfy the equation

$$
-X^{2}+Y^{3}-Z^{5} W+t^{2} Y Z^{3} W=0 .
$$

To get the affine equation of the singularity, we just put $W=1$; this is visibly an $E_{7}$ when $t \neq 0$. The coefficients of this polynomial depend on $t^{2}$ rather than just $t$. In this case, the reason is that the $\mathbf{5 6}$ denotes two 1/2-hypermultiplets. 


\section{Matter from the geometry of the vanishing 2-cycles}

In this section, we note how the matter representations can also be inferred from the geometry of the vanishing 2-cycles. We illustrate with a single example, although the method works for any configuration of vanishing 2-cycles corresponding to an A-D-E singularity.

Starting with an embedded $U(1)$, the positive roots which are neutral with respect to the $U(1)$ correspond to vanishing 2-cycles for $t \neq 0$. For $t=0$, we get more vanishing 2 -cycles. We can reverse this reasoning to start with the geometry of the vanishing 2-cycles and infer the $U(1)$, hence infer the matter content.

As our example, in [9], it was noted that the Calabi-Yau threefold $X_{5}$ arising from resolving singularities of $\mathbf{P}(1,1,2,6,8)[18]$ has families of vanishing 2-cycles which sweep out two divisors (called $D_{8}, D_{9}$ in [9]). For special parameter values, one of the vanishing 2-cycles split into a pair of vanishing 2-cycles.

Choosing $t$ to be a parameter for the vanishing 2-cycles with $t=0$ at a special parameter value, we name the vanishing 2-cycles at $t=0$ as $C_{1}, C_{2}, C_{3}$, with $C_{1}$ lying in $D_{8}$ and $C_{2}, C_{3}$ lying in $D_{9}$. To yield the desired neutrality of $C_{1}$ and $C_{2}+C_{3}$, we take the $U(1)$ in the direction given by $t_{1}=t_{2}=t_{4}$ in the $A_{3}$ Cartan. This gives $S U(3) \times U(1) \subset S U(4)$, and we predict the $\mathbf{3}$ of $S U(3)$, as was noted in the context of type IIA compactifications in [9] based on [8].

We would like to thank James Cogdell, Michael Bershadsky, Ken Intriligator, Shamit Kachru, Vladimir Sadov and David Morrison for helpful conversations. C.V. would also like to thank the hospitality of theory division at CERN where this work was completed. The research of S.K. was supported in part by NSF grant DMS-9311386 and NSA grant MDA904-96-1-0021. The research of C.V. was supported in part by NSF grant PHY-9218167. 


\section{References}

[1] M. Bershadsky, K. Intriligator, S. Kachru, D.R. Morrison, V. Sadov, C. Vafa, Geometric Singularities and Enhanced Gauge Symmetries, hep-th/9605200.

[2] D.R. Morrison and C. Vafa, Compactifications of F-Theory on Calabi-Yau ThreefoldsI,II, hep-th/9602114,9603161.

[3] E. Witten, to appear.

[4] C. Vafa, Evidence for F-theory, HUTP-96/A004, hep-th/9602022.

[5] P. Aspinwall and M. Gross, The SO(32) Heterotic String on a K3 surface, CLNS96/1409, hep-th/9605131.

[6] S. Katz, D. Morrison and R. Plesser, Enhanced Gauge Symmetry in Type II String Theory, hep-th/9601108.

[7] S. Katz and D.R. Morrison, Jour. Alg. Geom. 1 (1992) 449.

[8] M. Bershadsky, V. Sadov, and C. Vafa, D-strings on D-manifolds, HUTP-95/A035, hep-th/9510225.

[9] P. Berglund, S. Katz, A. Klemm, P. Mayr, New Higgs Transitions between Dual N $=2$ String Models, NSF-ITP-95-162, hep-th/9605154. 